\title{
PET and MR Imaging: The Odd Couple or a Match Made in Heaven?
}

\author{
Ciprian Catana*, Alexander R. Guimaraes*, and Bruce R. Rosen
}

Athinoula A. Martinos Center for Biomedical Imaging, Department of Radiology, Massachusetts General Hospital and Harvard Medical School, Charlestown, Massachusetts

\begin{abstract}
PET and MR imaging are modalities routinely used for clinical and research applications. Integrated scanners capable of acquiring PET and MR imaging data in the same session, sequentially or simultaneously, have recently become available for human use. In this article, we describe some of the technical advances that allowed the development of human PET/MR scanners; briefly discuss methodologic challenges and opportunities provided by this novel technology; and present potential oncologic, cardiac, and neuropsychiatric applications. These examples range from studies that might immediately benefit from PET/MR to more advanced applications on which future development might have an even broader impact.
\end{abstract}

Key Words: multimodality imaging; PET/MR imaging; applications; oncology; neurology; cardiology

J Nucl Med 2013; 54:815-824

DOI: 10.2967/jnumed.112.112771

$\mathbf{P}_{\mathrm{B}}$ ET is a quantitative technique that provides exceptionally sensitive assays of a wide range of biologic processes, allowing the detection of low concentrations of molecules of interest labeled with positron emitters. However, it suffers from lesser spatial resolution, in many cases has limited anatomic information, and involves ionizing radiation. MR provides high-resolution anatomic information with excellent soft-tissue contrast and the ability to measure a variety of physiologic, metabolic, and biochemical parameters. On the other hand, the molar sensitivity of MR for different metabolites and probes is many orders of magnitude lower than that of PET, imposing significant restrictions on the kinds of targets that can be visualized. Furthermore, absolute quantification of substrate concentration with MR remains challenging. Given the complementary nature of each modality's strengths and weaknesses, integrating PET and MR imaging offers the opportunity to gain in a single

\footnotetext{
Received Aug. 15, 2012; revision accepted Nov. 26, 2012.

For correspondence or reprints contact: Ciprian Catana, A.A. Martinos

Center, Building 149, 13th St., Room 2.301, Charlestown, MA 02129.

E-mail: ccatana@nmr.mgh.harvard.edu

Published online Mar. 14, 2013.

${ }^{*}$ Contributed equally to this work.

COPYRIGHT () 2013 by the Society of Nuclear Medicine and Molecular Imaging, Inc.
}

examination many of the positive attributes of both and mitigate some of their limitations. The wealth of information provided by MR enables PET/MR to go far beyond simple anatomic registration of PET molecular imaging, and the simultaneous acquisition of PET and MR data opens opportunities impossible to realize using sequentially acquired data.

This article paraphrases the Wagner Lecture delivered by Bruce Rosen at the 2011 Society of Nuclear Medicine Annual Meeting and summarizes the technical aspects of PET/MR imaging in addition to emphasizing the areas in which it may make a significant impact. Although these areas include oncologic, neurologic, cardiac, and psychiatric applications, PET/MR imaging has the potential to have a dramatic impact on the burgeoning field of molecular imaging.

\section{WHY NOW?-TECHNICAL ASPECTS}

Although PET/CT scanners have quickly become wellestablished clinical tools (1), the development of combined PET and MR imaging has been much slower because of numerous technical challenges on both sides.

The major obstacle to performing PET in or near an MR scanner is the presence of the magnetic field, which causes gain changes and spatial distortion in photomultiplier tubes, the scintillation light detector of choice for PET scanners. The fundamental technical advance that has made simultaneous PET/MR possible for clinical use was the emergence of a new type of solid-state photon detector (i.e., the avalanche photodiode), which maintains the light sensitivity of photomultiplier tubes while being insensitive to magnetic fields (2). More recently, another type of magnetic field-insensitive silicon-based photon-detector-called the silicon photomultiplier-has been proposed for developing an integrated PET/MR system $(3,4)$. Based on avalanche photodiode technology but operated in Geiger mode (allowing high timing resolution), these devices hold great promise for becoming the photon detector of choice in the PET/ MR field.

PET, in turn, can be problematic for MR imaging. For example, image artifacts or a decreased signal-to-noise ratio can be caused by electromagnetic interference; eddy currents can be induced in the PET shielding materials; and homogeneity of the constant magnetic field, $\mathrm{B}_{0}$, can be 
disrupted by susceptibility effects due to PET components. Though all issues are important, perhaps the most significant challenge until recently has been the limited space available inside the bore of standard MR systems. Gradient systems in particular pay a steep price and performance penalty from increased size, as power requirements go steeply with radius, and manufacturing tolerances for gradient shielding become much more demanding. For many years, the widest-bore MR imaging systems were no larger than $60 \mathrm{~cm}$ in diameter, providing no additional space for integrating key PET components. However, recently new gradient designs have allowed peak performance with larger, 70-cm, bore diameters, providing (just) enough space for the PET camera.

Beyond these 2 technical advances (i.e., development of magnetic field-insensitive PET photon detectors and largerbore magnets), another major factor setting the stage for clinical PET/MR was the success of PET/CT, which has proven the value and clinical relevance of combining anatomic and molecular information during a single scanning session.

\section{Integrated Scanners for Human Use}

For the first decade after simultaneous PET and MR data acquisition had first been demonstrated in vivo in small animals (5), most of the progress in the field was made in the preclinical arena.

Fortunately, the major medical equipment manufacturers have realized the potential of this emerging field, and the first integrated scanner for human brain imaging was installed in 2007. This prototype PET insert into an MR scanner, called BrainPET (Siemens Healthcare, Inc.) (Fig. 1A), was integrated with a standard 3-T MR scanner (Magnetom TIM Trio; Siemens Healthcare, Inc.) and proof-of-principle simultaneous data acquisition was demonstrated (6-8). When not in use, the BrainPET can be docked at the back of the magnet, without obstructing the bore so that the MR scanner can be used in stand-alone mode.

Quickly on the heels of this development, Philips developed a whole-body sequential PET/MR imaging scanner (Ingenuity TF) (Fig. 1B) addressing the challenges of the magnetic field and space limitations of MR by placing the PET scanner adjacent to the MR scanner (the 2 scanners are

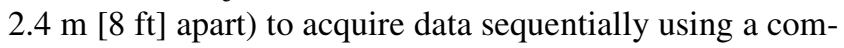
mon patient table, similarly to PET/CT scanners (9). One advantage of this approach is that the state-of-the-art timeof-flight PET scanner (Gemini TF PET; Philips) is modified so that the PET detectors work in the vicinity of the MR scanner and a separate MR imaging (Achieva 3.0T X-series; Philips) system is used. However, simultaneous data acquisition is not possible using this approach. This scanner received the CE mark in Europe and Food and Drug Administration 510(k) clearance in the United States.

GE Healthcare has also begun to explore the sequential approach and designed a new table to shuttle patients between the 2 scanners and compatible with both. In this approach, GE Healthcare uses its own state-of-the-art timeof-flight PET/CT scanner (Discovery PET/CT 690) and a 3-T MR scanner (Discovery MR750) located in adjacent rooms.

Recently, Siemens introduced a fully integrated wholebody PET/MR scanner, the Biograph mMR (Fig. 1C). Similar to the BrainPET prototype, the Biograph mMR uses avalanche photodiode technology but places the PET detectors in the space between the gradient coils and the radiofrequency body coil, using the additional bore space of a more advanced gradient design. In this way, the 2 scanners have been fully integrated and the resulting $60-\mathrm{cm}-$ diameter bore size allows for whole-body simultaneous PET/MR imaging (10). This scanner also received the CE mark in Europe and $510(\mathrm{k})$ clearance from the Food and Drug Administration in United States.

From here on, we will use the term PET/MR to refer to both sequential and simultaneous PET/MR, especially when describing common challenges or applications that would benefit from both approaches. The word simultaneous will be used when the distinct advantages offered by the temporal correlation of the measured signals are highlighted.

\section{Technical Challenges and Opportunities}

PET/MR imaging provides distinct challenges, and opportunities, when compared with PET/CT. One, attenuation

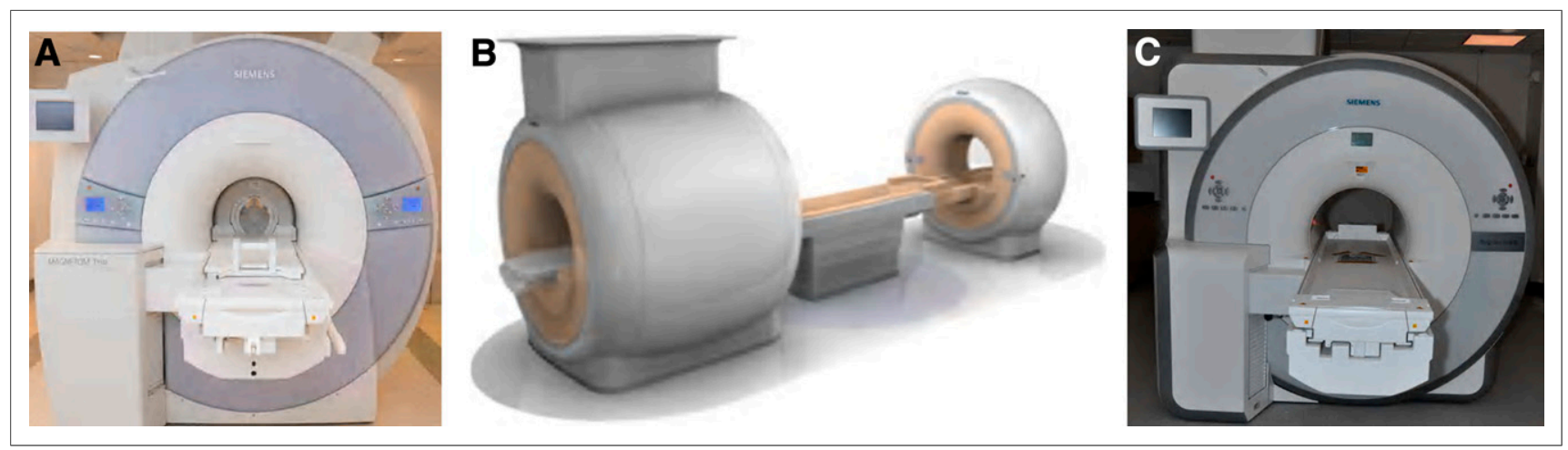

FIGURE 1. Integrated PET/MR scanners currently available for human use: (A) Siemens BrainPET/MR prototype, (B) Philips sequential $\mathrm{PET} / \mathrm{MR}$ whole-body scanner, and (C) Siemens Biograph mMR whole-body scanner. 
correction, immediately presents itself as a problem for any system without an ionizing radiation source or CT scanner. A second, the capability for dynamic motion correction, presents itself as a unique opportunity in simultaneous PET/ MR systems. Indeed, sometimes tackling one set of challenges leads to other opportunities; solving the problem of attenuation and motion correction would potentially allow for improved attenuation correction in simultaneous PET/ MR relative to PET/CT since misregistration of attenuation maps with the PET emission data can be fully mitigated. There are of course other relevant technical and practical issues (e.g., setting up a PET/MR facility (11) and designing combined data acquisition protocols (12)) that will not be discussed in this review.

Because of technical difficulties in placing and operating a rotating transmission source inside the MR scanner bore or room and the limited space available, the MR data have to be used for deriving the attenuation maps in the integrated scanners developed to date. Several factors have to be considered in order to implement an accurate MR-based method to account for the photon attenuation caused by the subject and the hardware located in the PET field of view (e.g., radiofrequency coils).

Although the MR soft-tissue contrast offers many ways to infer tissue type, a particularly challenging task consists of differentiating bone tissue from air-filled spaces, both of which appear as signal voids on the MR images obtained using conventional pulse sequences. This is the worst possible outcome, as bone is especially relevant as a photonattenuating medium, being the tissue with the highest linear attenuation coefficient. Atlas-based methods have been implemented for deriving the attenuation map from the MR data (13-15), and these have proven quite useful, although they can potentially lead to errors in patients with modified anatomy. One interesting alternative is the use of so-called ultra-short echo time sequences, previously developed for imaging cortical bone and other connective tissues $(16,17)$. These sequences are used to image solidphase tissues with short $\mathrm{T} 2$ relaxation times such as bone (T2's in bone typically range from 0.05 to $2 \mathrm{~ms}$, compared with relaxation times of $50-100 \mathrm{~ms}$ for most other soft tissues). Methods for generating the head attenuation maps from ultra-short echo time data have been implemented recently $(7,18,19)$. Extending these methods to the whole body remains challenging but is an area of active research. As initial solutions, methods in which linear attenuation coefficients corresponding to soft tissue are assigned to bone voxels have been proposed by the major manufacturers $(20,21)$. Good-quality images were obtained using these methods, though the quantitative properties of these data still need to be evaluated.

PET studies are usually long, and subject motion is difficult to avoid, leading to degradation (blurring) of PET images and to severe artifacts when motion has large amplitude, offsetting the benefit of using high-resolution scanners. Although other methods for minimizing or tracking the subject's motion have been proposed with variable success, in a simultaneous PET/MR imaging scanner, estimates of high-temporal-resolution motion can be derived from the MR data and used for rigid-body (22) and non-rigid-body (23-25) PET motion correction.

Although many challenges still remain, MR-assisted PET motion correction could dramatically reduce the spatial blurring and artifacts associated with PET movement of solid organs. If techniques to track the motion in the background of the sequences used for acquiring standard MR data are successfully developed, this unique opportunity enabled by simultaneous PET/MR could completely revolutionize the way PET is performed for certain applications (e.g., neurologic, lung, liver, and cardiac imaging).

\section{WHY NOW?-POTENTIAL APPLICATIONS}

Although PET/CT and stand-alone MR are independently useful imaging modalities, there are numerous unmet medical needs that may benefit from this new hybrid technology. We now highlight some of these, starting with clinical situations in which the benefits of PET/MR systems are most apparent and moving to applications on which future development might have even broader impact.

\section{Low-Hanging Fruit: Studies in Which PET/MR May Have Immediate Impact}

Patients for Whom Radiation Exposure Is a Concern. ${ }^{18} \mathrm{~F}$-FDG PET/CT has improved diagnostic accuracy for most pediatric malignancies (26-28). Furthermore, studies have suggested that ${ }^{18} \mathrm{~F}$-FDG uptake is a compelling and early surrogate marker of treatment efficacy in various pediatric malignancies $(29,30)$. Yet, to date, these studies are limited, and although prospective trials are needed, they may be driven by the increasing consideration of radiation risk to the pediatric population $(28,31,32)$ because whenever a PET scan is needed in children, a CT scan is also required for attenuation correction or anatomic correlation. However, the radiation dose being delivered to the patient is of special concern in this population for at least 2 reasons. First, the effective dose from CT is several times higher in newborns and children than in adults for the same acquisition settings (33). Second, pediatric patients have a higher lifetime risk of developing cancer relative to adults (34). MR in PET/MR devices could replace CT for attenuation correction in these patients, reducing the radiation dose by at least 50\% compared with a PET/CT study. Furthermore, collecting the data simultaneously limits anesthetic times for the pediatric populations who benefit from both examinations.

Similarly, radiation exposure is of concern in patient populations in need of multiple PET/CT scans, such as lymphoma patients who require PET/CT for staging at the time of diagnosis, during therapy, and at the end of treatment. However, this repeated scanning comes with significant exposure to ionizing radiation, on the order of $23-26 \mathrm{mSv}$, with the PET component contributing just 5-7 mSv of this 
dose. Thus, there is interest in an alternative imaging modality, such as MR imaging, that provides accurate anatomic localization and functional imaging without the associated excess radiation exposure of CT scanning.

Areas of the Body Where CT Is Suboptimal for Discriminating Anatomy. In the head and neck area, MR imaging is superior to CT in terms of accurate staging of tumor extent, involvement of vital soft-tissue structures, and nodal involvement $(35,36)$. Because of the added soft-tissue discrimination capability of MR imaging, PET/MR imaging will likely improve the assessment of tumor extent, involvement of bony structures, and bone marrow. Posttreatment surveillance presents many challenges because tissue distortion, scarring, and fibrosis from radiation and surgery can obscure early detection of recurrence by conventional follow-up. At our institution, approximately $20 \%$ of patients with malignancies of the head and neck require both ${ }^{18}$ F-FDG PET/CT and MR imaging in order to marry the soft-tissue discrimination of MR imaging with the metabolic specificity of ${ }^{18} \mathrm{~F}$-FDG PET.

MR imaging is the method of choice for evaluating pelvic malignancies (i.e., gynecologic, rectal (Fig. 2), and prostatic cancers), because of its improved soft-tissue discrimination compared with CT. For example, in cervical (Fig. 3) and endometrial cancers, MR imaging plays a principal role in staging in terms of parametrial invasion (T staging). However, locoregional control (discrimination of lymph node metastases) is not fully achieved in most cases. Although ovarian cancers are rare, benign adnexal masses (functional cysts, endometriosis, infectious processes, nonmalignant growths) are common; thus, it is challenging to identify those lesions that will benefit from surgical resection while sparing patients from the morbidity of unnecessary surgery. MR imaging has demonstrated improved potential based on morphology alone and T1 and T2 signal intensity characteristics. In all these cases, combining the MR data with PET will likely improve the accuracy of clinical staging.

MR imaging is also preferred for staging prostate cancer because of its accuracy at diagnosing extracapsular extent and neural invasion and its ability to incorporate multiple specific biomarkers that have shown promise in diagnosing prostate cancer (e.g., dynamic contrast-enhanced MR imaging, diffusion-weighted imaging, and MR spectroscopy). Yet, its sensitivity is approximately only $80 \%$ for the primary malignancy and often becomes much lower when bony metastases outside the pelvis, and lymph node metastases, are being considered. A recent study showed that the combination of MR imaging with ${ }^{11} \mathrm{C}$-acetate PET/ CT was superior to the individual methods alone for detecting localized prostate cancer (37). Furthermore, a different study suggested that combining ${ }^{11} \mathrm{C}$-choline and apparent diffusion coefficient measurements improved the tissueto-background contrast of disease with a Gleason score of $\geq 3+4$ (38). Thus, morphologic and potentially multiparametric MR imaging may be the modality of choice for providing anatomic and physiologic correlation to the PET findings, improving the accuracy with which primary tumor (Fig. 4) and distant metastases are assessed (39).

Areas in Which MR Imaging Offers Improved Tissue Specificity. In breast cancer, MR imaging has proven useful for local staging and treatment monitoring and has sensitivity greater even than conventional imaging methods (i.e., x-ray mammography and sonography). On the other hand, its specificity is variable and might be improved when combined with spatially fused ${ }^{18} \mathrm{~F}$-FDG PET images (40). Several factors, however, limit the efficacy of spatially registering the images acquired from stand-alone MR imaging and PET systems, and near-perfect spatial coregistration requires simultaneous acquisition. Furthermore, axillary lymph node status, which is the most powerful prognostic indicator in breast cancer patients, can be better assessed
FIGURE 2. Simultaneous PET/MR examination in colorectal cancer patient. T1-weighted postcontrast MR image demonstrates enhancing mass within rectum (arrow). PET image shows ${ }^{18} \mathrm{~F}-\mathrm{FDG}$ avidity of mass without anatomic correlate. Diffusion-weighted image (DWI) at same level demonstrates hyperintense lesion. Low signal intensity compatible with restricted diffusion is observed in apparent diffusion coefficient (ADC) map. Data were acquired on a Biograph mMR scanner at A.A. Martinos Center, Massachusetts General Hospital.

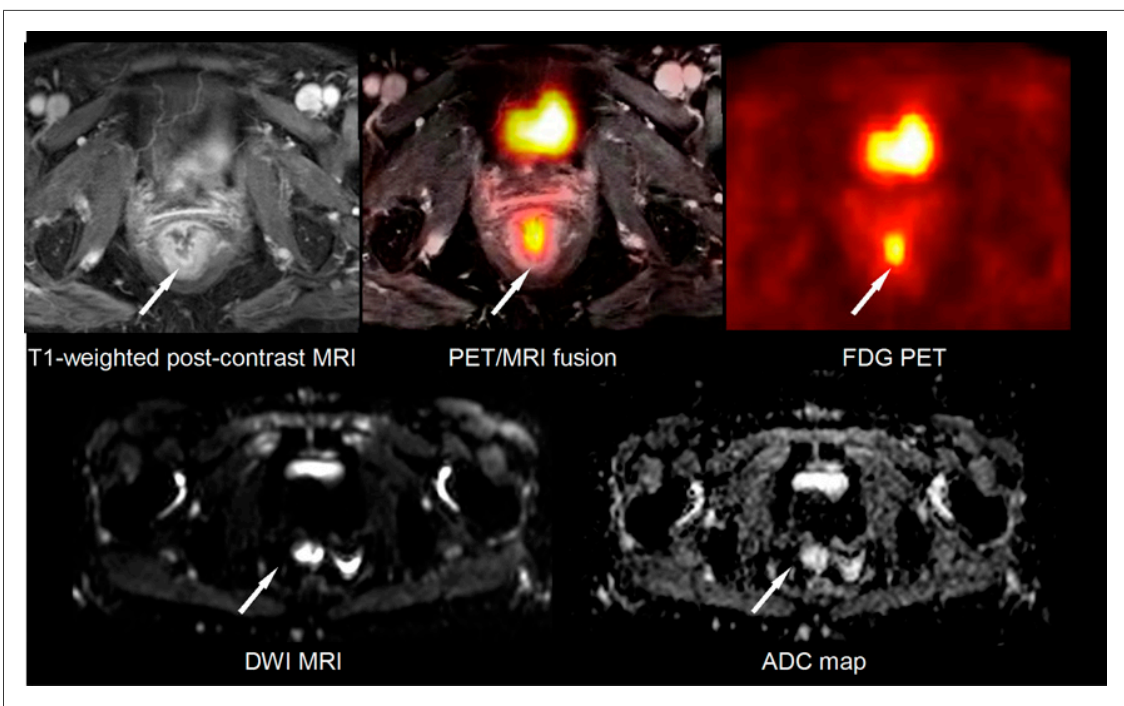




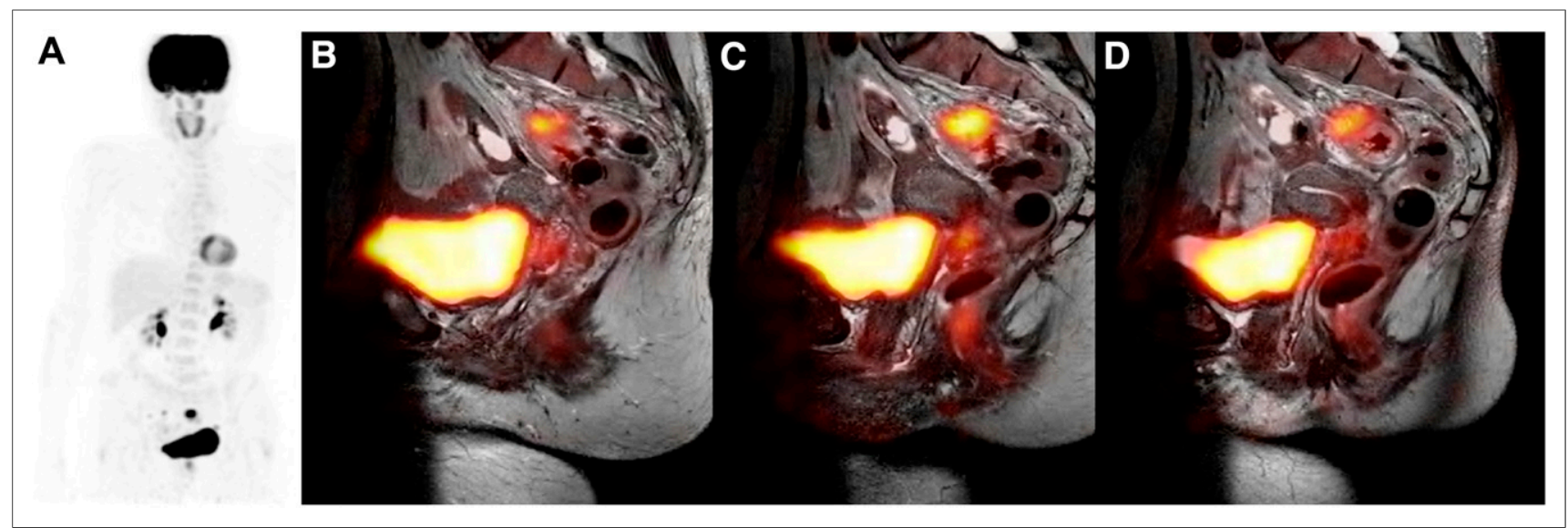

FIGURE 3. Sequential PET/MR for staging in patient with epidermoid carcinoma of cervix after conization and sigmoidectomy. (A) Wholebody PET shows hypermetabolic uptake in lower pelvis. (B-D) MR imaging shows thickening of colon wall with involvement of outer fatty tissue corresponding to hypermetabolic tracer uptake. Follow-up biopsy revealed granulomatosis without residual tumor. Data were acquired on a Philips Ingenuity PET/MR scanner at University Hospital of Geneva (courtesy of Osman Ratib).

with PET/MR using ${ }^{18} \mathrm{~F}-\mathrm{FDG}$ or other radiolabeled targeted agents and MR imaging lymph node-specific agents.

The liver is a common site for distant metastases from many subtypes of cancers. MR imaging has been shown to be useful and superior in its ability to discriminate small lesions (e.g., $<10 \mathrm{~mm})(41) .{ }^{18} \mathrm{~F}-\mathrm{FDG}$ PET remains problematic because of the heterogeneous uptake in the normal liver, its low sensitivity to lesions smaller than $10 \mathrm{~mm}$, and the concomitant decrease in sensitivity in patients with underlying liver disease, including cirrhosis and nonalcoholic fatty liver disease (42). PET/MR imaging will likely play a large role in determining the true sensitivity of ${ }^{18} \mathrm{~F}-\mathrm{FDG}$ PET as compared with other liver-specific reticuloendothelial or hepatocytespecific agents for determining the true extent of disease.

Bone marrow involvement is one of the most important prognostic factors in patients with lymphoma. In a metaanalysis conducted by $\mathrm{Wu}$ et al., PET/CT was demonstrated to be superior to MR imaging or PET alone in the staging of lymphoma (43). However, this study came under scrutiny, and it was suggested that prospective studies for comparing the distinct (and complementary) value of each imaging modality in specific settings are required (44). Multiple myeloma (Fig. 5) is the most frequent primary neoplasm of the skeletal system. Whole-body MR imaging has been proposed for detecting infiltrative focal bone marrow lesions and was demonstrated to have higher sensitivity than a skeletal survey for this task. Compared with the radiation exposure of $\mathrm{PET} / \mathrm{CT}$, that of PET/MR imaging would be minimized. Furthermore, as novel agents having demonstrated sensitivity in multiple myeloma or novel therapies are developed (e.g., bortezomib), PET/MR imaging may be the perfect tool to test these strategies.

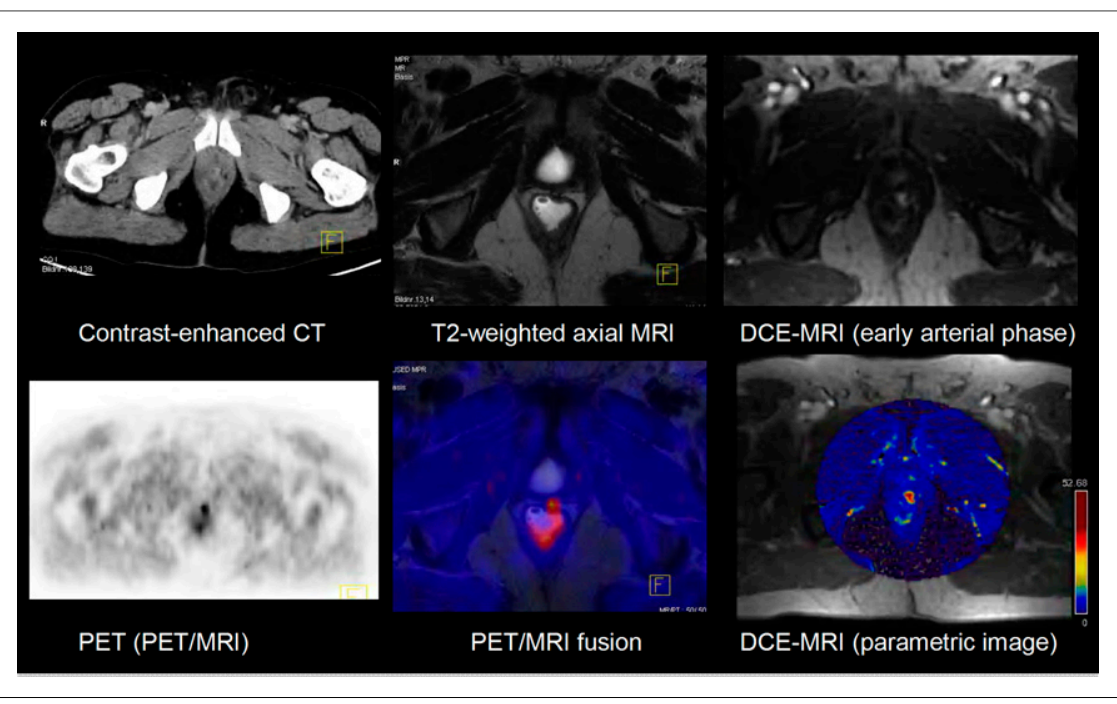

FIGURE 4. Multiparametric imaging using ${ }^{11} \mathrm{C}$-choline PET and dynamic contrast-enhanced (DCE) MR imaging increases diagnostic confidence and accuracy in patient with prostate-specific antigen recurrence after radical prostatectomy. Only faint anatomic correlate is observed in contrast-enhanced CT. Similarly, T2-weighted MR image shows superb anatomic detail but only small tumor correlate. However, clear enhancement is observed in early arterial phase of dynamic contrast-enhanced MR imaging and parametric map, correlating with PET signal. Cumulative evidence suggesting local recurrence as opposed to scar tissue or unspecific enhancement increases diagnostic certainty. Data were acquired on Biograph mMR scanner at Technische Universität München/Ludwig-Maximilians-Universität, Munich, Germany (courtesy of Ambros Beer). 


\section{Advanced Physiologic Applications: The Next Generation of PET/MR Studies}

Treatment Monitoring in Oncology. A growing understanding of the underlying molecular biology of cancer has led to the development of novel therapies targeting various molecular pathways active in cancer. Unlike the conventional cytotoxic chemotherapeutic agents, many of the molecularly targeted agents are cytostatic, causing inhibition of tumor growth rather than tumor regression. In this context, conventional endpoints such as tumor volume reduction may be delayed as compared with other metabolic or physiologic parameters. Combined PET/MR studies may provide important biomarkers to predict and monitor targeted treatment response and to document pharmacodynamic response.

The emerging importance of angiogenesis as a cancer therapy target makes assays of vascularity important to clinical research and future clinical practice related to targeted cancer therapy. Dynamic contrast-enhanced MR imaging allows the assessment of tumor vascularity and detects changes associated with angiogenesis-targeted therapy (45-47). For example, in glioblastoma patients treated with vascular endothelial growth factor inhibitors, evidence of tumor vascular normalization was demonstrated using dynamic contrast-enhanced MR imaging (48). However, when only dynamic contrast-enhanced MR imaging is used, the true antitumor effects of these agents cannot be completely understood, and combining PET parameters (e.g., estimates of tumor glucose metabolism, cellular proliferation, and amino-acid transport) and MR imaging methods may provide a better approach to this investigation. Furthermore, alternative probing of the microvascular system with magnetic nanoparticles has shown promise at interrogating antivascular effects in preclinical models (49).

Combined PET/MR measurements might help quantify precisely how tumor vascular properties (assessed by functional MR methods), proliferation, and antitumor effects (assessed with PET) occur and interact. First, a richer dataset is obtained using both imaging modalities (Fig. 6).
Second, the quantification of PET might be improved using the simultaneously acquired MR information (e.g., MRassisted PET motion and partial-volume-effect correction, MR-based radiotracer arterial input function estimation). Third, the meaning of PET findings might be better understood using MR information. All these tools might enable a more precise understanding of tumor biology and therapeutic response, both for trials of new treatment protocols and perhaps even on an individual basis. The wide range of responses among patients suggests that further studies of individual responses to therapy, correlating structural and functional imaging, metabolic imaging, and clinical findings (e.g., survival), will be helpful in understanding the mechanism of action of novel therapeutic agents.

Cardiac Applications. In the clinical assessment of patients with cardiovascular disease, PET allows quantification of blood flow and is considered the gold standard for assessing myocardial tissue viability. MR can inform about ventricle function; structural changes; and, using contrast agents, perfusion and tissue viability. It was suggested that the combination of these methods in a simultaneous PET/ MR scanner (Fig. 7) allows a more detailed risk assessment to be performed (50). Furthermore, non-rigid-body MRassisted motion correction methods have the potential to significantly improve PET data quantification and reproducibility.

Both PET and MR imaging are also used for cardiovascular applications in molecular imaging. In the context of monitoring stem cell therapy, direct labeling either with ${ }^{18} \mathrm{~F}-\mathrm{FDG}$ or gadolinium and magnetic nanoparticles and reporter gene approaches allow the noninvasive imaging of stem cells, and some of these methods have already been used in clinical trials (51). PET/MR could improve the short-term assessment of stem cell delivery and the longterm treatment efficacy.

Several groups have recently started to develop duallabeled PET/MR probes. For example, magnetic nanoparticles coupled to chelated ${ }^{64} \mathrm{Cu}$ have been proposed for targeting vascular inflammation (52) and tumor integrin

FIGURE 5. Simultaneous PET/MR imaging of myeloma patient. (A) Plain radiograph of left knee demonstrating lytic lesions in medial femoral condyle and proximal tibia (arrows). (B-D) Coronal ${ }^{18} \mathrm{~F}-\mathrm{FDG}$ PET (B), fat-saturated T2-weighted MR (C), and fused (D) images at same level demonstrating concordant foci in right tibia, right femoral condyle, and left medial femoral condyle. Data were acquired on a Biograph mMR scanner at A.A. Martinos Center, Massachusetts General Hospital.

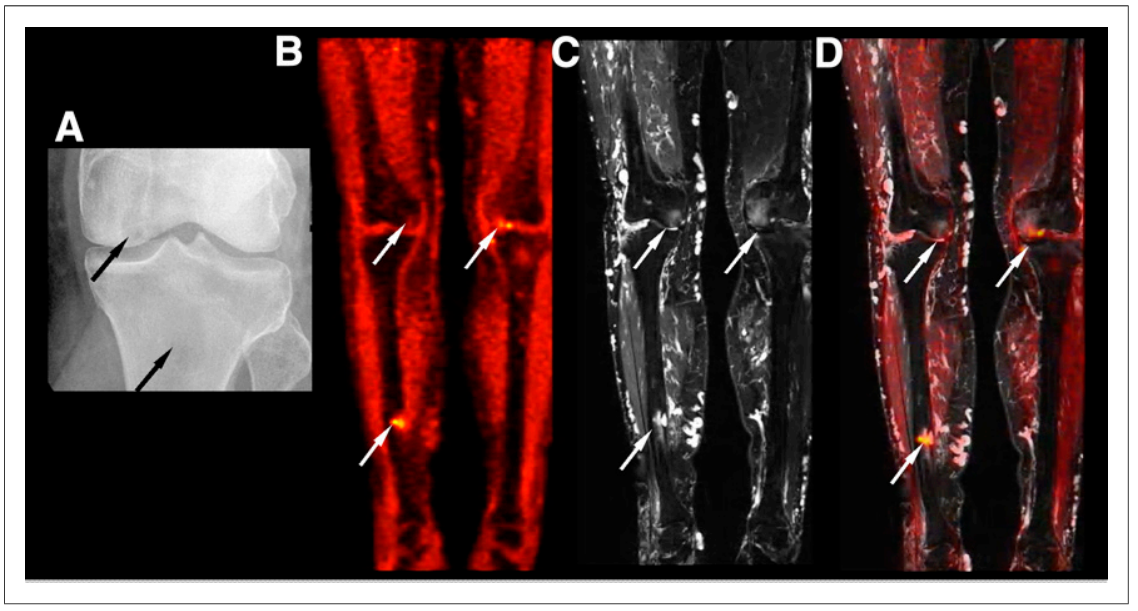




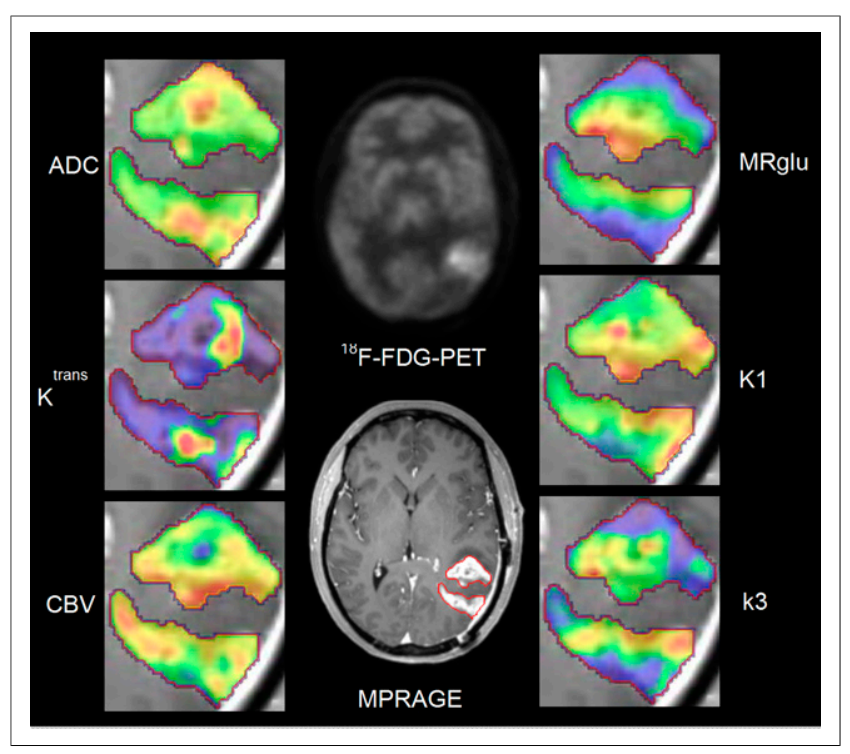

FIGURE 6. Multiparametric PET/MR imaging of glioblastoma: ${ }^{18} \mathrm{~F}-$ FDG PET and morphologic magnetization-prepared rapid gradient echo (MPRAGE) MR image after administration of MR contrast agent (middle column). Parameters derived from MR data (vascular permeability [ $\left.\mathrm{K}^{\text {trans }}\right]$, cerebral blood volume [CBV], and apparent diffusion coefficient [ADC]) and PET data (metabolic rate of glucose [MRglu], $K_{1}$, and $k_{3}$ ) in region of interest (red contour) defined on enhancing part of tumor are shown in left and right columns, respectively. Data were acquired on a BrainPET/MR prototype at A.A. Martinos Center, Massachusetts General Hospital (courtesy of Dan Chonde and Dominique Jennings).

$\alpha_{v} \beta_{3}$ expression (53). In our center, we have focused on atherosclerotic plaque imaging as a potential first application for bimodal probes (54).

\section{Future Applications: Potential Domains in Which PET/ MR May Change How We Practice}

Neuropsychiatric Diseases. The burden from neuropsychiatric disorders (expressed as disability-adjusted life years lost) is higher than the burden from any other disease category in the developed world (55), yet despite the amazing advances in brain imaging over the last $30 \mathrm{y}$, imaging has had little to no impact on today's clinical practice (56). Nevertheless, both PET and MR imaging have had a profound impact on our understanding of neuropsychiatric diseases, from an improved understanding of neurotransmitter imbalances in schizophrenia to an evolving understanding of brain network perturbations in diseases such as depression and autism (57). Indeed, a comprehensive understanding of psychiatric diseases must encompass the integration of neurochemical, genetic, behavioral, and circuit-based models. Today the dominant tools for these investigations, at the brain level, are PET and MR imaging; thus, their marriage becomes a natural one for the study of mental illnesses. Although simultaneous PET/MR systems are thus likely to be profoundly useful for translational investigations, might this tool find its way into clinical practice?

Perhaps the first application will be in the study of patients with suspected Alzheimer disease. The evolving understanding of this disease as one encompassing a potentially long prodromal state (58) preceding definitive clinical manifestations, and the forthcoming arrival of disease-modifying treatments, will likely require both an earlier and a more definitive diagnosis. In this regard, PET and MR imaging provide complementary information (Fig. 8) in the assessment of Alzheimer disease patients (59-61), with PET's ability to characterize amyloid (and soon $\tau$-protein) buildup regionally, and MR's ability to see the associated neuronal degeneration and changes in circuit behavior (62). Imaging strategies in the future thus will likely extend beyond today's "rule out" with MR imaging alone (to exclude other organic causes of dementialike tumor or hydrocephalus) to comprehensive "rule-in" studies, assessing amyloid or $\tau$-burden (through improved PET quantification facilitated by MR-assisted PET motion and partial-volume-effect correction) and their sequelae in terms of direct observation of ongoing neuronal degeneration and cortical dysfunction. Given the likely expense, and potential morbidity, associated with therapy-altering treatments, the combined use of PET/MR may provide a cost-effective way to assess who should, and should not, enter into such therapeutic regimens.

Beyond Alzheimer disease, the crystal ball is cloudier but with no less potential for impact. Today, as novel treatments for disorders such as medically intractable depression are being explored (63), the need for pretherapeutic diagnoses to match the precision of these treatments will present itself. Indeed, Mayberg (63) used both PET and MR for the pre- and posttreatment evaluation of her patients, and it is likely that the combination of modalities will emerge alongside these new therapeutic approaches to provide the

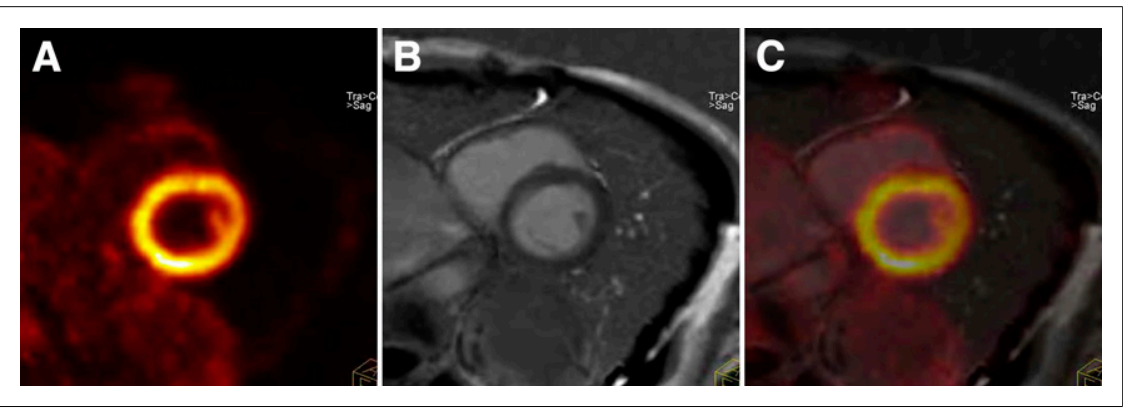

FIGURE 7. Simultaneous cardiac PET/MR study. (A and B) Electrocardiograph-gated PET (A) and delayed contrast-enhanced cardiac MR (B) images. PET data were acquired in list mode and binned. (C) MR images acquired in diastole and fused with diastolic PET data. Patient has normal heart. Data were acquired on a Biograph mMR scanner at Washington University in St. Louis (courtesy of Pamela Woodard and Richard Laforest). 
pharmacologic and physiologic information required to make informed treatment decisions. One tool that may emerge as a key asset in our exploration of therapeutic options for psychiatric diseases is the combination of PET and MR to study the dynamics of neurotransmission. PET investigations for the last $20 \mathrm{y}$ have certainly provided the foundation for these studies, but the simultaneous collection of functional MR data should allow for important advances in these methods, both through the clearer definition of associated networks and through the concurrent collection of neurophysiologic parameters to refine (perhaps redefine) the traditional kinetic models used to analyze such PET data.

Lymph Node Imaging. Surgical assessment is the gold standard for the diagnosis of lymph node metastases. However, surgical lymphadenectomy confers an increased risk of immediate and delayed complications, and noninvasive techniques that accurately identify lymph node metastases are needed.

${ }^{18} \mathrm{~F}-\mathrm{FDG}$ PET/CT has played a role in evaluating lymph node metastases of multiple oncologic etiologies but has demonstrated mixed sensitivities and specificities. MR imaging using contrast agents administered interstitially or intravenously has been proposed as an alternative. Ultra-small superparamagnetic iron oxide (also known as magnetic nanoparticles)-enhanced MR imaging is the method that has shown the most promise and has been used for $\mathrm{N}$ staging of patients with head and neck (64), breast (65), stomach (66), and prostate (67) cancer. Although ultra-small superparamagnetic iron oxide agents are not yet approved for clinical use and further trials are needed to demonstrate their utility, recent provocative evidence has been shown with other Food and Drug Administration-approved MR imaging contrast agents (68).

Several studies investigated the relationship between MR and PET measurements for the assessment of metastatic lymph nodes. For example, a statistically significant inverse correlation was observed between apparent diffusion coefficient values and standardized uptake values (SUVs) in metastatic lymph nodes of head and neck squamous cell carcinoma (69). In non-small cell lung cancer, short-inversion-time inversion-recovery turbo spin-echo MR imaging proved more accurate than diffusion-weighted MR imaging and ${ }^{18} \mathrm{~F}$-FDG PET/CT (70). PET/CT is thought to have a higher sensitivity and specificity to detect retroperitoneal lymph node metastasis than do current cross-sectional imaging modalities. Although these preliminary results suggest a complementary role for PET and MR imaging in the evaluation of lymph nodes, these studies would likely benefit from the perfect spatial coregistration and improved PET data quantification (e.g., partial-volume-effect correction) provided by a simultaneous PET/MR scanner.

Beyond ${ }^{18} \mathrm{~F}-\mathrm{FDG}$ in Oncology. ${ }^{18} \mathrm{~F}-\mathrm{FDG}$ is currently used with PET for primary staging, assessment of treatment response, and follow-up in more than $90 \%$ of cancers (71). However, because of various factors (e.g., variable expression of hexokinase and glucose transporters in hepatocellular carcinoma (72)), not all tumors show a significant increase in metabolic activity on ${ }^{18} \mathrm{~F}$-FDG PET imaging, and other tracers have entered clinical trials (73). In the context of drug development, PET allows the characterization of the pharmacokinetics and pharmacodynamics of novel agents that can be radiolabeled with positron emitters.

A PET/MR scanner may provide an ideal tool for testing and validating these agents. First, the reduced radiation exposure when compared with CT will facilitate the translation to human studies. Second, MR imaging provides the anatomic details needed for assessing the whole-body distribution of these tracers. Third, the potential for improved data quantification will allow researchers to go beyond semiquantitative methods (e.g., the standardized uptake value). Finally, advanced MR techniques will provide physiologic information complementary to the PET data, including basic physiologic and biophysical measurements and the mapping of endogenous metabolites with MR spectroscopy.

In Vivo Quantification of Smart MR Probes. MR contrast agents induce relaxation of tissue water, and the extent of this relaxation enhancement, termed relaxivity, depends on several factors that influence the accessibility of water to the MR active agent (paramagnetic ion or superparamagnetic nanoparticle), as well as the overall concentration of the contrast agent. In a seminal paper, Louie et al. demonstrated that the relaxivity of a specifically designed contrast agent could be changed in the presence of the enzyme $\beta$-galactosidase (74). Numerous publications have followed that described smart agents responsive to other enzymes, $\mathrm{pH}$, partial pressure of oxygen, and temperature, among other factors. However, a key limitation of these

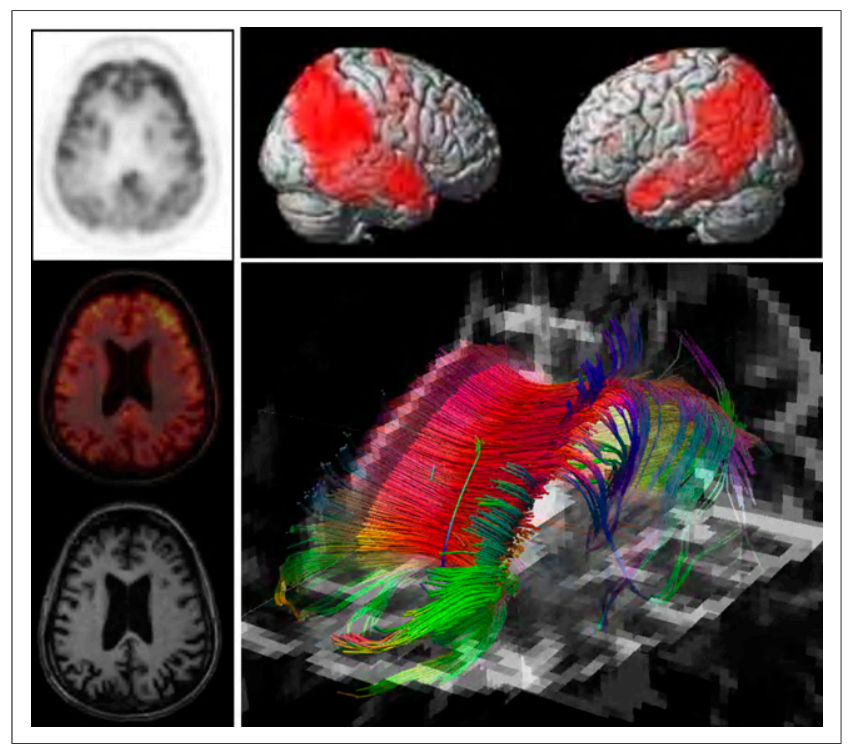

FIGURE 8. Simultaneous PET/MR study of Alzheimer disease patient. Left column: axial ${ }^{18} \mathrm{~F}-\mathrm{FDG}$ PET, morphologic MR, and fused images. Top right: Surface projections of cerebral metabolism showing areas with reduced metabolism as compared with controls. Bottom right: Diffusion tensor imaging showing white matter tracts in same patient. Data were acquired on a BrainPET/MR prototype at A.A. Martinos Center, Massachusetts General Hospital. 
approaches is that the MR signal depends on both the relaxivity of the contrast agent and the local probe concentration-typically the product of these 2 factors. In vivo, the probe concentration is in general unknown, will change with time, and may vary in diseased versus normal tissue. Using a bimodal PET/MR probe, it has proven to be possible to use PET for estimating the overall concentration and the MR data to determine the molar relaxivity of the agent, allowing for determination of its specific biochemical or physiologic target (75).

\section{CONCLUSION}

Despite the technical demands underlying the matchmaking between PET and MR, the differences between these 2 partners is the greatest source of strength in their potential marriage. Odd though the underlying technologies may be to each other, finding ways to bring these 2 pillars of medical imaging together can confer a high degree of synergy. And like any good marriage, the more intimate the connection between them, the stronger the resulting partnership. Many factors will decide the ultimate role of PET/MR systems within our overall health care system, not the least of which is the cost of such systems, and the degree to which the benefits accrued match the resources required to perform and interpret these studies in the clinic. Training the next generation of interpreters in the art and science of both PET and MR is another challenge that will have to be met if this tool is to have widespread impact outside a small group of academic sites. Nevertheless, if the future of clinical practice is precision medicine, where therapeutic decisions are designed around specific molecular pathologic events at the earliest possible stage, then PET/MR systems may be the first of the next generation of molecular imaging tools for that future.

\section{DISCLOSURE}

The costs of publication of this article were defrayed in part by the payment of page charges. Therefore, and solely to indicate this fact, this article is hereby marked "advertisement" in accordance with 18 USC section 1734. No potential conflict of interest relevant to this article was reported.

\section{REFERENCES}

1. Townsend DW, Carney JP, Yap JT, Hall NC. PET/CT today and tomorrow. J Nucl Med. 2004;45(suppl 1):4S-14S

2. Pichler B, Lorenz E, Mirzoyan R, et al. Performance test of a LSO-APD PET module in a 9.4 Tesla magnet. Paper presented at: 1997 IEEE Nuclear Science Symposium; November 9-15, 1997; Albuquerque, NM.

3. Kolb A, Lorenz E, Judenhofer MS, Renker D, Lankes K, Pichler BJ. Evaluation of Geiger-mode APDs for PET block detector designs. Phys Med Biol. 2010; 55:1815-1832.

4. Seong Jong H, In Chan S, Ito M, et al. An investigation into the use of Geigermode solid-state photomultipliers for simultaneous PET and MRI acquisition. IEEE Trans Nucl Sci. 2008;55:882-888.
5. Shao Y, Cherry SR, Farahani K, et al. Simultaneous PET and MR imaging. Phys Med Biol. 1997;42:1965-1970.

6. Schlemmer H-PW, Pichler BJ, Schmand M, et al. Simultaneous MR/PET imaging of the human brain: feasibility study. Radiology. 2008;248:1028-1035.

7. Catana C, van der Kouwe A, Benner T, et al. Toward implementing an MRIbased PET attenuation-correction method for neurologic studies on the MR-PET brain prototype. J Nucl Med. 2010;51:1431-1438.

8. Herzog H, Langen KJ, Weirich $\mathrm{C}$, et al. High resolution BrainPET combined with simultaneous MRI. Nuklearmedizin. 2011;50:74-82.

9. Zaidi H, Ojha N, Morich M, et al. Design and performance evaluation of a whole-body Ingenuity TF PET/MRI system. Phys Med Biol. 2011;56:3091-3106.

10. Delso G, Furst S, Jakoby B, et al. Performance measurements of the Siemens mMR integrated whole-body PET/MR scanner. J Nucl Med. 2011;52:1914-1922.

11. Sattler B, Jochimsen T, Barthel H, et al. Physical and organizational provision for installation, regulatory requirements and implementation of a simultaneous hybrid PET/MR-imaging system in an integrated research and clinical setting. MAGMA. 2013;26:159-171.

12. Martinez-Möller A, Eiber M, Nekolla SG, et al. Workflow and scan protocol considerations for integrated whole-body PET/MRI in oncology. J Nucl Med. 2012;53:1415-1426.

13. Montandon ML, Zaidi H. Atlas-guided non-uniform attenuation correction in cerebral 3D PET imaging. Neuroimage. 2005;25:278-286.

14. Hofmann M, Steinke F, Scheel V, et al. MRI-based attenuation correction for PET/MRI: a novel approach combining pattern recognition and atlas registration. J Nucl Med. 2008;49:1875-1883.

15. Rota Kops E, Herzog H. Template-based attenuation correction of PET in hybrid MR-PET scanners [abstract]. J Nucl Med. 2008;49(suppl):162P.

16. Reichert ILH, Robson MD, Gatehouse PD, et al. Magnetic resonance imaging of cortical bone with ultrashort TE pulse sequences. Magn Reson Imaging. 2005; 23:611-618.

17. Robson MD, Bydder GM. Clinical ultrashort echo time imaging of bone and other connective tissues. NMR Biomed. 2006;19:765-780.

18. Keereman V, Vandenberghe S, De Deene Y, Luypaert R, Broux T. MR-based attenuation correction for PET using an Ultrashort Echo Time (UTE) sequence. Nuclear Science Symposium Conference Record. Piscataway, NJ: IEEE; 2008: 4656-4661.

19. Berker Y, Franke J, Salomon A, et al. MRI-based attenuation correction for hybrid PET/MRI systems: a 4-class tissue segmentation technique using a combined ultrashort-echo-time/Dixon MRI sequence. J Nucl Med. 2012;53:796-804.

20. Martinez-Möller A, Souvatzoglou M, Delso G, et al. Tissue classification as a potential approach for attenuation correction in whole-body PET/MRI: evaluation with PET/CT data. J Nucl Med. 2009;50:520-526.

21. Schulz V, Torres-Espallardo I, Renisch S, et al. Automatic, three-segment, MRbased attenuation correction for whole-body PET/MR data. Eur J Nucl Med Mol Imaging. 2011;38:138-152.

22. Catana C, Benner T, van der Kouwe A, et al. MRI-assisted PET motion correction for neurologic studies in an integrated MR-PET scanner. J Nucl Med. 2011; 52:154-161.

23. Chun SY, Reese TG, Ouyang J, et al. MRI-based nonrigid motion correction in simultaneous PET/MRI. J Nucl Med. 2012;53:1284-1291.

24. Dikaios N, Izquierdo-Garcia D, Graves MJ, Mani V, Fayad ZA, Fryer TD. MRIbased motion correction of thoracic PET: initial comparison of acquisition protocols and correction strategies suitable for simultaneous PET/MRI systems. Eur Radiol. 2012;22:439-446.

25. King AP, Buerger C, Tsoumpas C, Marsden PK, Schaeffter T. Thoracic respiratory motion estimation from MRI using a statistical model and a 2-D image navigator. Med Image Anal. 2012;16:252-264.

26. Franzius C. FDG-PET/CT in pediatric solid tumors. Q J Nucl Med Mol Imaging. 2010;54:401-410.

27. McCarville MB. PET-CT imaging in pediatric oncology. Cancer Imaging. 2009;9:35-43.

28. Voss SD. Pediatric oncology and the future of oncological imaging. Pediatr Radiol. 2011;41(suppl 1):S172-S185.

29. Furth C, Steffen IG, Amthauer H, et al. Early and late therapy response assessment with ${ }^{18} \mathrm{~F}$ fluorodeoxyglucose positron emission tomography in pediatric Hodgkin's lymphoma: analysis of a prospective multicenter trial. J Clin Oncol. 2009;27:4385-4391.

30. Hawkins DS, Conrad EU, Butrynski JE, Schuetze SM, Eary JF. F-18-fluorodeoxy-D-glucose-positron emission tomography response is associated with outcome for extremity osteosarcoma in children and young adults. Cancer. 2009; 115:3519-3525.

31. Alessio AM, Kinahan PE, Manchanda V, Ghioni V, Aldape L, Parisi MT. Weight-based, low-dose pediatric whole-body PET/CT protocols. J Nucl Med. 2009;50:1570-1577. 
32. Robbins E. Radiation risks from imaging studies in children with cancer. Pediatr Blood Cancer. 2008;51:453-457.

33. Jadvar H, Connolly LP, Fahey FH, Shulkin BL. PET and PET/CT in pediatric oncology. Semin Nucl Med. 2007;37:316-331.

34. Fahey FH. Dosimetry of pediatric PET/CT. J Nucl Med. 2009;50:1483-1491.

35. Fruehwald-Pallamar J, Czerny C, Mayerhoefer ME, et al. Functional imaging in head and neck squamous cell carcinoma: correlation of PET/CT and diffusionweighted imaging at 3 Tesla. Eur J Nucl Med Mol Imaging. 2011;38:1009-1019.

36. Yoon DY, Hwang HS, Chang SK, et al. CT, MR, US, ${ }^{18}$ F-FDG PET/CT, and their combined use for the assessment of cervical lymph node metastases in squamous cell carcinoma of the head and neck. Eur Radiol. 2009;19:634-642.

37. Jambor I, Borra R, Kemppainen J, et al. Improved detection of localized prostate cancer using co-registered MRI and ${ }^{11} \mathrm{C}$-acetate PET/CT. Eur J Radiol. 2012; 81:2966-2972.

38. Park H, Wood D, Hussain H, et al. Introducing parametric fusion PET/MRI of primary prostate cancer. J Nucl Med. 2012;53:546-551.

39. Beer AJ, Eiber M, Souvatzoglou M, Schwaiger M, Krause BJ. Radionuclide and hybrid imaging of recurrent prostate cancer. Lancet Oncol. 2011;12:181-191.

40. Moy L, Ponzo F, Noz ME, et al. Improving specificity of breast MRI using prone PET and fused MRI and PET 3D volume datasets. J Nucl Med. 2007;48: 528-537.

41. Niekel MC, Bipat S, Stoker J. Diagnostic imaging of colorectal liver metastases with CT, MR imaging, FDG PET, and/or FDG PET/CT: a meta-analysis of prospective studies including patients who have not previously undergone treatment. Radiology. 2010;257:674-684.

42. Floriani I, Torri V, Rulli E, et al. Performance of imaging modalities in diagnosis of liver metastases from colorectal cancer: a systematic review and meta-analysis. J Magn Reson Imaging. 2010;31:19-31.

43. Wu L-M, Chen F-Y, Jiang X-X, Gu H-Y, Yin Y, Xu J-R. ${ }^{18}$ F-FDG PET, combined FDG-PET/CT and MRI for evaluation of bone marrow infiltration in staging of lymphoma: a systematic review and meta-analysis. Eur J Radiol. 2012; $81: 303-311$

44. Kwee TC, Nievelstein RAJ. Is MRI less accurate than FDG-PET/CT in diagnosing bone marrow involvement in lymphoma? Eur J Radiol. 2011;80:565-566.

45. Rosen MA, Schnall MD. Dynamic contrast-enhanced magnetic resonance imaging for assessing tumor vascularity and vascular effects of targeted therapies in renal cell carcinoma. Clin Cancer Res. 2007;13(suppl):770S-776S.

46. Wedam SB, Low JA, Yang SX, et al. Antiangiogenic and antitumor effects of bevacizumab in patients with inflammatory and locally advanced breast cancer. J Clin Oncol. 2006;24:769-777.

47. Drevs J, Siegert P, Medinger M, et al. Phase I clinical study of AZD2171, an oral vascular endothelial growth factor signaling inhibitor, in patients with advanced solid tumors. J Clin Oncol. 2007;25:3045-3054.

48. Batchelor TT, Sorensen AG, di Tomaso E, et al. AZD2171, a pan-VEGF receptor tyrosine kinase inhibitor, normalizes tumor vasculature and alleviates edema in glioblastoma patients. Cancer Cell. 2007;11:83-95.

49. Guimaraes AR, Ross R, Figuereido JL, Waterman P, Weissleder R. MRI with magnetic nanoparticles monitors downstream anti-angiogenic effects of mTOR inhibition. Mol Imaging Biol. 2011;13:314-320.

50. Nekolla SG, Martinez-Moeller A, Saraste A. PET and MRI in cardiac imaging: from validation studies to integrated applications. Eur J Nucl Med Mol Imaging. 2009;36(suppl):S121-S130.

51. Zhang WY, Ebert AD, Narula J, Wu JC. Imaging cardiac stem cell therapy: translations to human clinical studies. J Cardiovasc Transl Res. 2011;4:514-522.

52. Jarrett BR, Gustafsson B, Kukis DL, Louie AY. Synthesis of Cu-64-labeled magnetic nanoparticles for multimodal imaging. Bioconjug Chem. 2008;19: 1496-1504.

53. Lee HY, Li Z, Chen K, et al. PET/MRI dual-modality tumor imaging using arginine-glycine-aspartic (RGD): conjugated radiolabeled iron oxide nanoparticles. J Nucl Med. 2008;49:1371-1379.

54. Uppal R, Catana C, Ay I, et al. Simultaneous MR-PET imaging of thrombus with a fibrin-targeted dual MR-PET probe: a feasibility study. Radiology. 2011; 258:812-820.
55. WHO. The global burden of disease: 2004 update. http://www.who.int/healthinfo/ global_burden_disease/GBD_report_2004update_part4.pdf.

56. Rosen BR, Savoy RL. fMRI at 20: has it changed the world? Neuroimage. 2012; 62:1316-1324.

57. Pina-Camacho L, Villero S, Fraguas D, et al. Autism spectrum disorder: does neuroimaging support the DSM-5 proposal for a symptom dyad? A systematic review of functional magnetic resonance imaging and diffusion tensor imaging studies. J Autism Dev Disord. 2012;42:1326-1341.

58. Amieva H, Le Goff M, Millet X, et al. Prodromal Alzheimer's disease: successive emergence of the clinical symptoms. Ann Neurol. 2008;64:492-498.

59. Buckner RL, Snyder AZ, Shannon BJ, et al. Molecular, structural, and functional characterization of Alzheimer's disease: evidence for a relationship between default activity, amyloid, and memory. J Neurosci. 2005;25:7709-7717.

60. Jack CR, Lowe VJ, Senjem ML, et al. C-II PiB and structural MRI provide complementary information in imaging of Alzheimers disease and amnestic mild cognitive impairment. Brain. 2008;131:665-680.

61. Villain N, Desgranges B, Viader F, et al. Relationships between hippocampal atrophy, white matter disruption, and gray matter hypometabolism in Alzheimer's disease. J Neurosci. 2008;28:6174-6181.

62. Sperling RA, Dickerson BC, Pihlajamaki M, et al. Functional alterations in memory networks in early Alzheimer's disease. Neuromolecular Med. 2010; $12: 27-43$

63. Mayberg HS. Targeted electrode-based modulation of neural circuits for depression. J Clin Invest. 2009;119:717-725.

64. Curvo-Semedo L, Diniz M, Miguéis J, et al. USPIO-enhanced magnetic resonance imaging for nodal staging in patients with head and neck cancer. J Magn Reson Imaging. 2006;24:123-131.

65. Memarsadeghi M, Riedl CC, Kaneider A, et al. Axillary lymph node metastases in patients with breast carcinomas: assessment with nonenhanced versus USPIOenhanced MR imaging. Radiology. 2006;241:367-377.

66. Tokuhara T, Tanigawa N, Matsuki M, et al. Evaluation of lymph node metastases in gastric cancer using magnetic resonance imaging with ultrasmall superparamagnetic iron oxide (USPIO): diagnostic performance in post-contrast images using new diagnostic criteria. Gastric Cancer. 2008;11:194-200.

67. Barentsz JO, Futterer JJ, Takahashi S. Use of ultrasmall superparamagnetic iron oxide in lymph node MR imaging in prostate cancer patients. Eur J Radiol. 2007;63:369-372.

68. Lambregts DMJ, Beets GL, Maas M, et al. Accuracy of gadofosveset-enhanced MRI for nodal staging and restaging in rectal cancer. Ann Surg. 2011;253:539-545.

69. Nakamatsu S, Matsusue E, Miyoshi H, Kakite S, Kaminou T, Ogawa T. Correlation of apparent diffusion coefficients measured by diffusion-weighted MR imaging and standardized uptake values from FDG PET/CT in metastatic neck lymph nodes of head and neck squamous cell carcinomas. Clin Imaging. 2012;36:90-97.

70. Ohno Y, Koyama H, Yoshikawa T, et al. N-stage disease in patients with nonsmall cell lung cancer: efficacy of quantitative and qualitative assessment with STIR turbo spin-echo imaging, diffusion-weighted MR imaging, and fluorodeoxyglucose PET/CT. Radiology. 2011;261:605-615.

71. Rice SL, Roney CA, Daumar P, Lewis JS. The next generation of positron emission tomography radiopharmaceuticals in oncology. Semin Nucl Med. 2011;41:265-282.

72. Paudyal B, Oriuchi N, Paudyal P, et al. Clinicopathological presentation of varying F-18-FDG uptake and expression of glucose transporter 1 and hexokinase II in cases of hepatocellular carcinoma and cholangiocellular carcinoma. Ann Nucl Med. 2008;22:83-86.

73. Caroli P, Nanni C, Rubello D, Alavi A, Fanti S. Non-FDG PET in the practice of oncology. Indian J Cancer. 2010;47:120-125.

74. Louie AY, Huber MM, Ahrens ET, et al. In vivo visualization of gene expression using magnetic resonance imaging. Nat Biotechnol. 2000;18:321-325.

75. Frullano L, Catana C, Benner T, Sherry AD, Caravan P. Bimodal MR-PET agent for quantitative pH imaging. Angew Chem Int Ed Engl. 2010;49:2382-2384 